\title{
Supernova Remnants and GLAST
}

\author{
Patrick Slane \\ Harvard-Smithsonian Center for Astrophysics
}

\begin{abstract}
It has long been speculated that supernova remnants represent a major source of cosmic rays in the Galaxy. Observations over the past decade have ceremoniously unveiled direct evidence of particle acceleration in SNRs to energies approaching the knee of the cosmic ray spectrum. Nonthermal X-ray emission from shell-type SNRs reveals multi-TeV electrons, and the dynamical properties of several SNRs point to efficient acceleration of ions. Observations of TeV gammaray emission have confirmed the presence of energetic particles in several remnants as well, but there remains considerable debate as to whether this emission originates with high energy electrons or ions. Equally uncertain are the exact conditions that lead to efficient particle acceleration.

Based on the catalog of EGRET sources, we know that there is a large population of Galactic gamma-ray sources whose distribution is similar to that of SNRs. With the increased resolution and sensitivity of GLAST, the gamma-ray SNRs from this population will be identified. Their detailed emission structure, along with their spectra, will provide the link between their environments and their spectra in other wavebands to constrain emission models and to potentially identify direct evidence of ion acceleration in SNRs. Here I summarize recent observational and theoretical work in the area of cosmic ray acceleration by SNRs, and discuss the contributions GLAST will bring to our understanding of this problem.
\end{abstract}

Keywords: GLAST; Supernova Remnants

PACS: $01.30 . \mathrm{Cc} ; 95.85 . \mathrm{Pw} ; 98.38 . \mathrm{Mz}$

\section{INTRODUCTION}

Supernova remnants represent an energetic class of objects that is intimately connected to sources of $\gamma$-ray emission in our Galaxy. Shock acceleration by the SNR blast wave provides ample energy for the production of multi-TeV particles, and the presence of nearby material in dense clouds from which the remnant progenitors collapsed forms a natural target for pion production with subsequent $\pi^{0} \rightarrow \gamma \gamma$ decay. Nonthermal bremsstrahlung of electrons off ambient material, as well as inverse Compton (IC) scattering of electrons off ambient photons, can also lead to $\gamma$-ray production - and in many cases are the dominant mechanisms. SNRs are thus strong candidates for the emission of $\gamma$-rays. Xray observations have revealed numerous remnants for which nonthermal emission from their shells provides direct evidence of $\mathrm{TeV}$ electrons. More recently, $\mathrm{TeV} \gamma$-ray emission has been detected from a number of SNRs, possibly providing evidence for acceleration of ions as well.

The EGRET source catalog contains a large number of unidentified sources in the Galactic plane, some of which have been tentatively associated with SNRs, and such associations are plausible - at least in principle; particularly for remnants that are expanding into dense environments, we expect emission that extends well into the EGRET band. However, the large positional uncertainties for these sources, along with the large sizes and high number density of SNRS, yield a relatively high probability for false identifications of EGRET sources with SNRs. Yet the emission in this band holds particular importance in helping to understand the emission at higher energies, and in addressing the overall picture of cosmic-ray acceleration in SNRs. Below I discuss the production of $\gamma$-ray emission from SNRs and describe recent results on evidence for cosmic-ray acceleration in SNRs, with particular emphasis on contributions that are expected from GLAST observations.

\section{PARTICLE ACCELERATION BY SNR SHOCKS}

As the blast wave from a supernova explosion expands, the surrounding circumstellar material (CSM) and interstellar medium (ISM) is swept up into a shell of hot gas. The shock jump conditions yield an increase in density by a factor of $n / n_{0}=(\gamma+1) /(\gamma-1)$, and the associated postshock temperature is given by

$$
T=\frac{2(\gamma-1)}{(\gamma+1)^{2}} \frac{\mu}{k} m_{p} V_{s}^{2}
$$



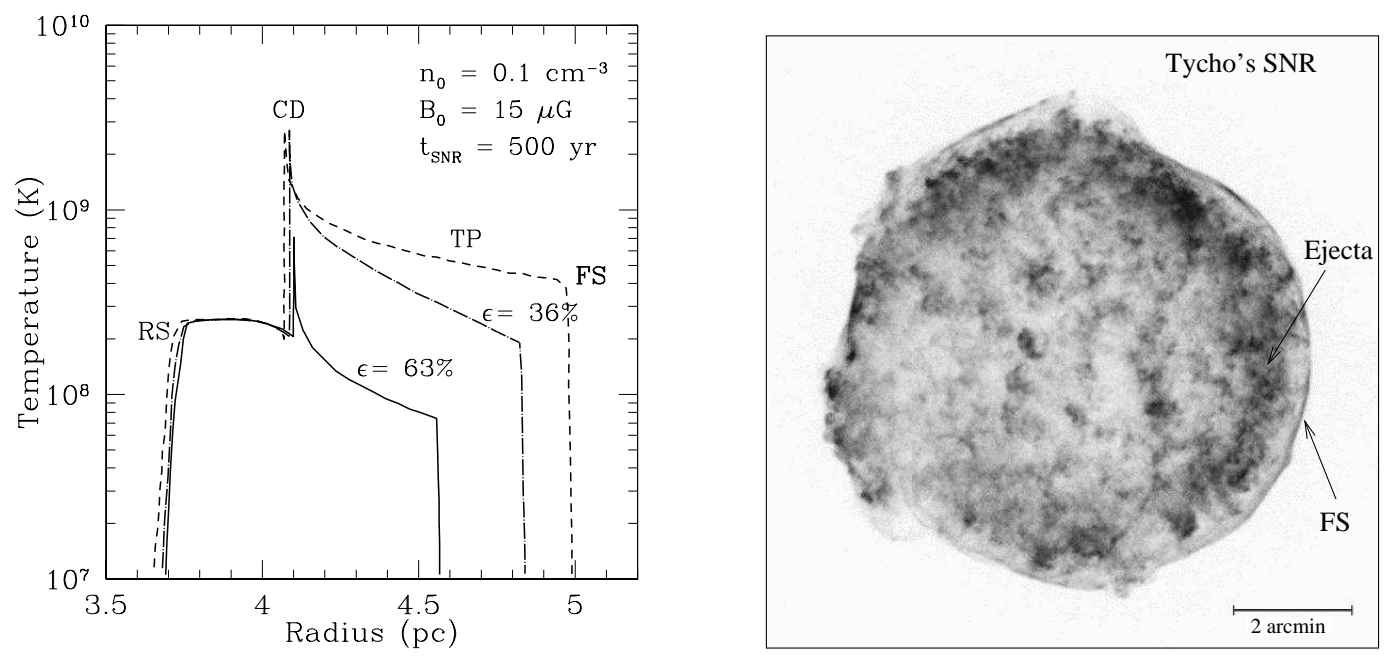

FIGURE 1. Left: SNR radial temperature distribution for different values of the particle acceleration efficiency. See text for description. Right: Chandra image of Tycho's SNR showing the thin rim of nonthermal emission at the forward shock (FS), and the inner shell of shocked ejecta.

where $\gamma$ is the adiabatic index for the gas ( $\gamma=5 / 3$ for an ideal gas), $V_{s}$ is the shock speed, $m_{p}$ is the proton mass, and $\mu$ is the mean molecular weight ( $\mu \approx 0.6$ for solar abundances). This shock-heated gas produces $\mathrm{X}$-ray emission characterized by a thermal bremsstrahlung spectrum accompanied by emission lines. As the blast wave decelerates upon sweeping up increasing amounts of material, a reverse shock propagates back into the ejecta. At early times, the X-ray spectrum is dominated by emission from the reverse-shocked ejecta; as the amount of swept-up material increases, the spectrum becomes dominated by emission from material with ISM abundances.

In addition to thermal heating of the swept-up gas, some fraction of the shock energy density goes into production of relativistic particles through diffusive shock acceleration wherein energetic particles streaming away from the shock form turbulent waves which act to scatter other particles back toward the shock. Subsequent reacceleration builds up a nonthermal population of high energy particles, with the maximum energy being limited by radiative losses, the age of the SNR, or particle escape. Such particle acceleration by SNR shocks has long been suggested as a process by which cosmic rays are produced, and radio observations provide direct evidence of nonthermal electrons with energies of several GeV.

If the relativistic particle component of the energy density becomes comparable to that of the thermal component, the shock acceleration process can become highly nonlinear. The gas becomes more compressible (with $\gamma$ becoming much larger than 5/3), which results in a higher density and enhanced acceleration. The resulting efficient production of nonthermal particles has a significant impact on the dynamical evolution of the shock. Figure 1 (left, from [1]) shows the SNR temperature as a function of radius at an age of $500 \mathrm{yr}$, assuming expansion into a uniform density $n_{0}=0.1 \mathrm{~cm}^{-3}$ with an ambient magnetic field strength $B_{0}=15 \mu \mathrm{G}$. The short-dashed curve (labeled TP) corresponds to the test-particle case in which no diffusive shock acceleration operates. The regions corresponding to the forward shock (FS), the reverse shock (RS) and the contact discontinuity (CD) that separates the swept-up and ejecta components are indicated. Also shown are temperature plots for cases of moderate $(\varepsilon=36 \%)$ and efficient ( $\varepsilon=63 \%$ ) particle acceleration. (Here $\varepsilon$ refers to the fraction of the energy flux crossing the shock that ends up in relativistic particles.) Two distinct observable effects are immediately evident: at a given age, the separation between the FS and the RS (or CD) decreases considerably with increased particle acceleration; and the temperature at the forward shock is reduced for cases of high acceleration efficiency.

\section{NONTHERMAL X-RAY EMISSION FROM SNRS}

While the shock-heated ejecta and CSM/ISM components of shell-type SNRs produce line-dominated X-ray spectra, as described above, a growing number of remnants also reveal evidence of hard, nonthermal X-ray emission components, apparently associated with high energy electrons accelerated by the SNR shock. In several cases - e.g., SN 1006 

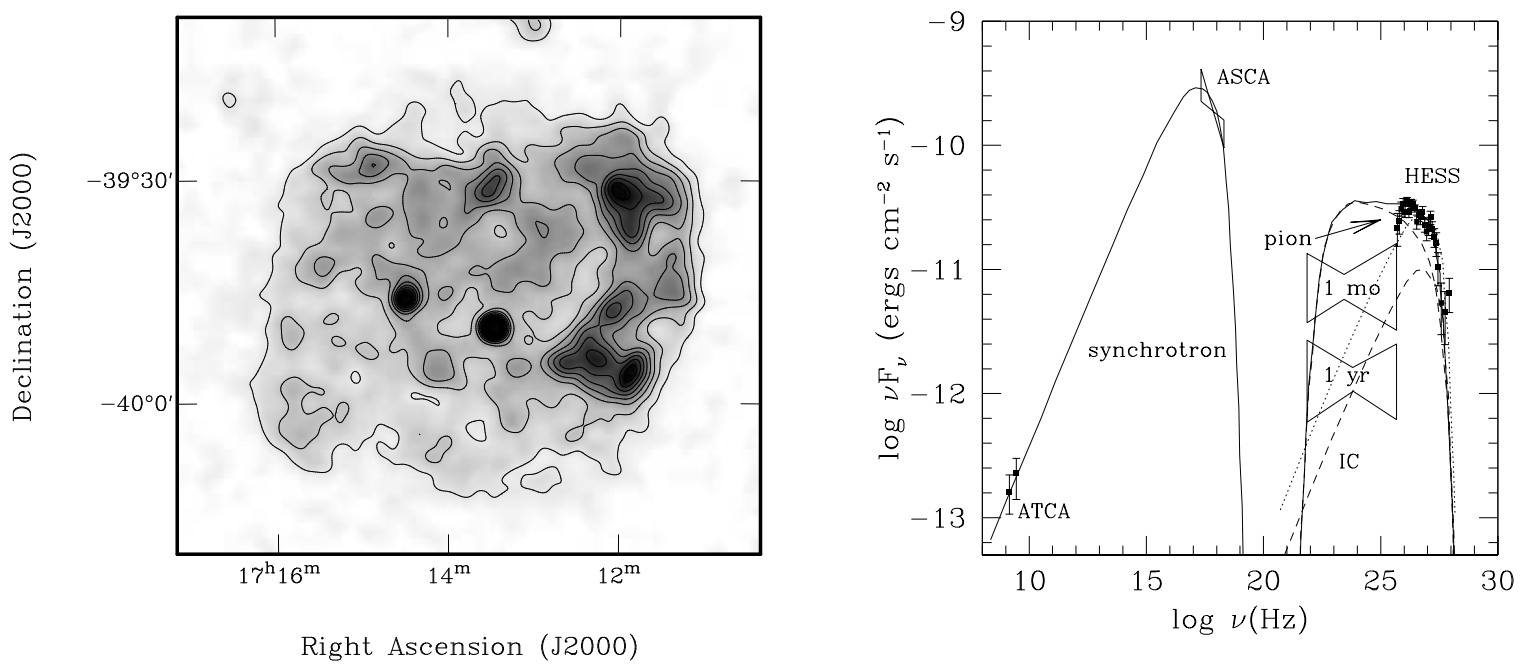

FIGURE 2. Left: ROSAT PSPC image of G347.3-0.5 (RX J1713.7-3946). Right: Broadband spectrum of G347.3-0.5. Models for the synchrotron, pion-decay, and inverse-Compton emission are described in the text. The GLAST sensitivity is indicated for 1 month and 1 year of all-sky survey data.

[2], G347.3-0.5 [3, 4], and G266.2-1.2 [5] - the nonthermal emission components completely dominate the thermal components, and the X-ray spectra from the shells are featureless. For others, including most of the known young SNRs - e.g., Cas A [6], Tycho [7], and Kepler [8] - thin rims of nonthermal emission surround the remnants directly along their forward shocks. These observations make it clear that SNRs are capable of accelerating electrons to very high energies. It is assumed that ions are accelerated by the same process although, as we describe below, the evidence for this is less direct. Since ions dominate the cosmic-ray energy density, however, it is exactly this evidence that is of particular importance for our understanding of cosmic ray production.

The Chandra image of Tycho's SNR is shown in Figure 1 (right). Spectral studies show that the thin outer rim is dominated by featureless emission consistent with synchrotron radiation from energetic electrons, while the brighter emission seen in a broad shell behind the rim is from shock-heated ejecta [9]. The position of the reverse shock is along the inner regions of this ejecta component while the contact discontinuity separating the ejecta and forward shock is located at the inner edge of the faint region separating the ejecta and the forward shock.

As discussed above, the separation between the forward shock and the contact discontinuity is strongly modified by the efficient acceleration of cosmic ray particles (Figure 1, left). In Tycho's SNR, this separation is considerably smaller than expected at the known age of the remnant unless a significant fraction of the explosion energy has gone into the acceleration of cosmic ray ions [9]. These measurements, along with evidence that the forward shock temperature in the young remnant 1E 0102.2-7219 is considerably lower than that expected from the measured expansion velocity [10] (see equation 1 above, which requires $\gamma \gg 5 / 3$ for the observed velocity to yield the temperature in this remnant), provide dynamical evidence for the acceleration of cosmic rays ions (which dominate the dynamics because of their large mass) by SNR shocks; despite the lack of a direct spectral signature (see below), these observations show that at least some SNRs accelerate ions, as well as electrons, to multi-TeV energies.

\section{GAMMA-RAY EMISSION MECHANISMS}

At higher particle energies, $\gamma$-ray production may result from nonthermal bremsstrahlung of electrons colliding with ambient gas, from IC scattering of ambient photons, and from the decay of neutral pions created by the collision of energetic protons. Without additional constraints, it can be difficult to differentiate between these mechanisms based on $\gamma$-ray spectra alone. For example, although pion-induced emission leads to a broad spectrum that, in general, can be distinguished from the more sharply-peak IC spectrum from a cut-off electron population (see Figure 2 and the discussion below), high magnetic fields can produce a steepening of the electron spectrum over a wide energy range, which can broaden the IC spectrum and make it difficult to differentiate from a pion-decay spectrum [1]. The relative contributions from the different $\gamma$-ray production mechanisms depend highly on ambient conditions, however, 

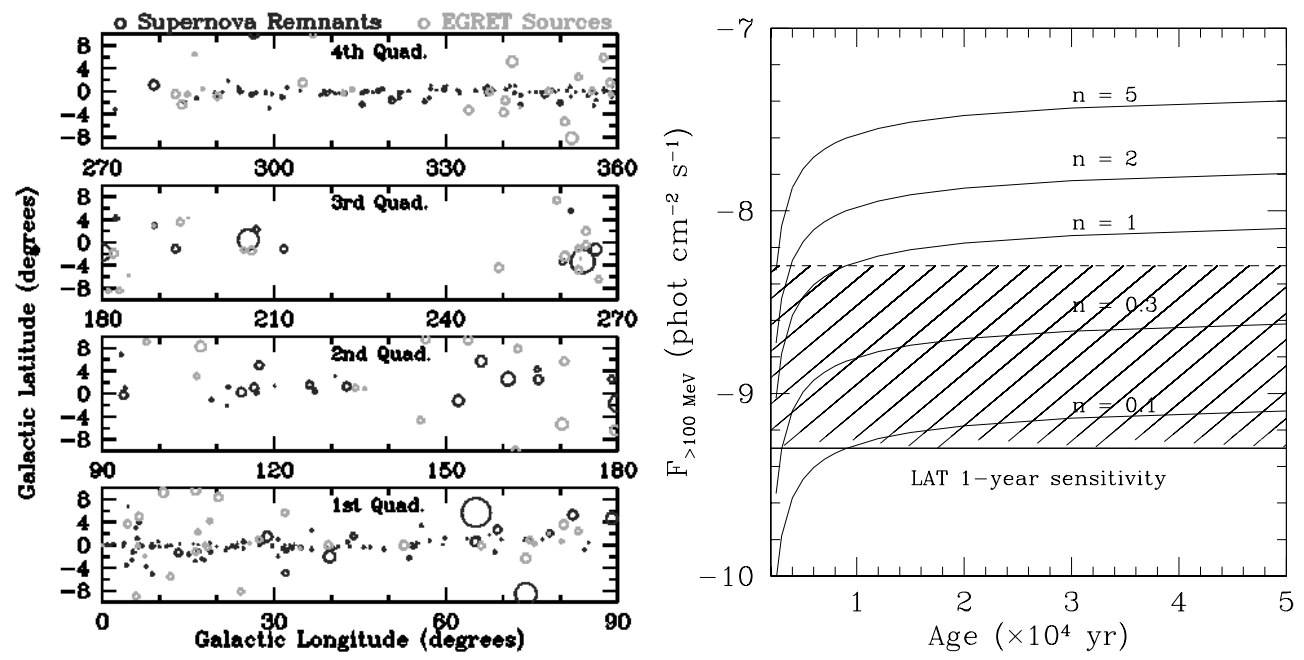

FIGURE 3. Left: Distribution of supernova remnants (black) and EGRET sources (grey) in the region of the Galactic plane. Circles indicate SNR sizes and EGRET source position uncertainties. Right: Predicted pion-decay $\gamma$-ray flux for SNR expansion into different densities. The solid horizontal line corresponds to the GLAST survey 1-year sensitivity for a point source. The sensitivity for extended emission such as that expected from SNRs depends on the size of the emission region; an arbitrary factor of 10 variation in sensitivity is illustrated for comparison with predicted fluxes.

and these conditions also have significant effects on the emission in other spectral bands. For a strong pion-decay component, for example, a reasonably high ambient density is required to provide ample target material with which energetic protons from the SNR shock can collide. Such dense material should also lead to significant thermal X-ray emission. A strong IC component, on the other hand, should be accompanied by synchrotron emission in the radio and $\mathrm{X}$-ray bands whose luminosity is consistent with expected values for the postshock magnetic field. In this sense, then, $\gamma$-ray measurements accompanied by spectra from other bands hold the best promise for interpreting the high energy emission - and, in particular, for providing spectral evidence of high energy ions.

ASCA observations of G347.3-0.5 (RX J1713.7-3946) revealed that the X-ray spectrum of this large-diameter SNR (Figure 2, left) is dominated by nonthermal emission [3, 4], indicating the presence of electrons with energies in excess of $\sim 10 \mathrm{TeV}$. Subsequent detection with HESS [11] confirmed the presence of energetic particles, but the nature of the $\gamma$-ray emission remains unclear. The broadband spectrum of the remnant is shown in Figure 3 (right) along with models for the emission. The radio and X-ray emission is well-described by synchrotron radiation from a power-law distribution of particles accompanied by a high energy cutoff. The high energy spectrum can be described by a model of emission induced by both protons (through pion decay) and electrons (through IC scattering), shown as dashed curves in Figure 2. Here the pion component dominates [12], although the assumed ambient density $\left(n_{0}=1 \mathrm{~cm}^{-2}\right)$ is hard to reconcile with upper limits derived from the lack of observed thermal X-ray emission from the remnant [4].

A larger overall normalization for the electron spectrum is able to adequately reproduce the $\mathrm{TeV}$ emission from G347.5-0.5 without any pion contribution (dotted curve in Figure 3), but this overpredicts the synchrotron emission unless a small filling factor is assumed for the magnetic field [13]. The GLAST sensitivity expected from all-sky survey mode exposures after 1 month and 1 year are indicated in Figure 2, and show that the pion component will either be detected or clearly ruled out within the first year of observations.

\section{GLAST AND SNRS}

The distribution of EGRET sources in the Galactic plane has considerable overlap with that of SNRs (Figure 3, left). Given the discussion above, this would seem reasonable; we expect to detect $\gamma$-rays from SNRs. However, the low angular resolution provided by EGRET results in large uncertainties for the source positions. The chance probability that some of these large error circles would overlap with the position of an SNR is relatively high, especially since the remnants themselves are extended. Moreover, the SNR population is closely linked to the distribution of pulsars and star-forming regions, both classes of which are potential $\gamma$-ray emitters. 
The expected pion-decay flux from a SNR is approximately

$$
F(>100 \mathrm{MeV}) \approx 4.4 \times 10^{7} \theta E_{51} d_{\mathrm{kpc}}^{-2} n_{0} \text { phot } \mathrm{cm}^{-2} \mathrm{~s}^{-1}
$$

where $\theta$ is a slow function of the SNR age, $E_{51}$ is the explosion energy in units of $10^{51} \mathrm{ergs}, d_{\mathrm{kpc}}$ is the distance in kpc, and $n_{0}$ is the preshock density [14]. For reasonable values of these parameters, the predicted fluxes are very near the sensitivity limit of EGRET. While the above estimates are conservative in that any contributions from IC scattering and bremsstrahlung have been ignored, it remains clear that only SNRs in particularly dense regions would be expected to produce detectable emission. Torres et al. [15] have investigated candidate associations of EGRET sources with SNRs for which there is some evidence for dense surrounding molecular material, and some of these associations are indeed plausible. But it is fair to say that there are no unambiguous detections of SNRs by EGRET.

This somewhat disconcerting view will receive a considerable boost from GLAST. In Figure 3 (right) we plot the estimated flux in the GLAST band as a function of SNR age, for a range of ambient densities. The solid horizontal line represents the LAT sensitivity for detection of a point source in one year of survey data. The sensitivity is a strong function of source extent; the hatched region shows a nominal factor of ten range in sensitivity to account for different SNR radii. While remnants in regions with densities typical of the ISM $\left(n_{0} \sim 0.3 \mathrm{~cm}^{-3}\right)$, appear unlikely to be detectable, there is a wide range of densities typical of those found in regions of massive star formation that should yield detectable SNR emission. For these sources, the prognosis is quite good: GLAST observations will provide spectra that probe the important region of the $\gamma$-ray spectrum than can differentiate between pion-decay and IC mechanisms, thus making a significant contribution to our understanding of particle acceleration in SNRs.

\section{SUMMARY}

Several mechanisms exist by which SNRs may be associated with the emission of energetic $\gamma$-rays. Recent X-ray and $\mathrm{TeV} \gamma$-ray observations have provided powerful evidence for the presence of high energy particles, and GLAST observations promise contributions in the crucial spectral range where pion-induced emission can potentially be discriminated from IC emission. The significant increase in angular resolution over that provided by EGRET will provide more confident associations between GLAST sources and SNRs, and the large collecting area will result in sufficient sensitivity to accurately determine spectra for comparison with broadband models. In addition, a significant increase in the discovery space of $\gamma$-ray emission from SNRs can be expected, allowing us to probe new associations and potentially resulting in GLAST detections that point the way to discoveries in other bands.

\section{ACKNOWLEDGMENTS}

I would like to acknowledge the collaborations on this subject that I have enjoyed with Don Ellison, Dan Patnaude, Steve Reynolds, Jasmina Lazendic, Jack Hughes, and Bryan Gaensler.

\section{REFERENCES}

1. D. C. Ellison et al. 2007, ApJ, accepted (astro-ph/0702674).

2. K. Koyama et al. 1995, Nature 378, 255.

3. K. Koyama et al. 1997, PASJ, 49, L7.

4. P. Slane et al. 1999, ApJ, 525, 357.

5. P. Slane et al. 2001, ApJ, 548, 814.

6. E. V. Gotthelf et al. 2001, ApJ, 552, L39.

7. U. Hwang et al. 2002, ApJ, 581, 1101.

8. A. Bamba et al. 2005, ApJ, 621, 793.

9. J. S. Warren et al. 2005, ApJ, 634, 376.

10. J. P. Hughes, C. E. Rakowski, \& A. Decourchelle 2000, ApJ, 543, L61.

11. F. Aharonian et al. 2006, A\&A, 449, 223.

12. K. Moraitis \& A. Mastichiadis 2007, A\&A, 462, 173.

13. J. S. Lazendic et al. 2004, ApJ, 602, 271.

14. L. O'C. Drury, F. A. Aharonian, \& H. J. Völk 1994, A\&A, 297, 959.

15. D. F. Torres et al. 2003, PhR, 382, 303. 